\title{
Behaviour of reinforcement in drop tower beam tests
}

\author{
Lena Leicht ${ }^{1, *}$, Franz Bracklow ${ }^{1}$, Marcus Hering ${ }^{1}$, and Manfred Curbach $^{1}$ \\ ${ }^{1}$ Technische Universität Dresden, Institute of Concrete Structures, Germany
}

\begin{abstract}
Drop tower tests help to gain understanding about the general behaviour of reinforced concrete members under impact loading and to analyse strains and strain rates occurring within their reinforcement. For this purpose, beam and slab specimens are usually employed. The main advantage of beams compared to slabs is that they are less complex due to the almost two-dimensional instead of three-dimensional wave propagation within them. To investigate the steel strains and strain rates, ten impact tests on beam specimens with various impact energies were performed. The impactor sizes and velocities were varied. The reinforcement bars of the beams were instrumented with semiconductor strain gauges. The measured data suggest that the occurring strains in beam tests are independent of the loading velocity. The same was found for the strain rates. The reason is that higher impact energies mostly influence the concrete damage due to spalling on the impact-facing side which happens after the maximum strains occurred. The strains in the reinforcement bars generally result from the overall deflection because of the impact, the spreading of longitudinal waves in the horizontal direction, and the localized cracking of the concrete due to the formation of a punching cone.
\end{abstract}

\section{Impact tests on concrete beams}

Many researchers conducted beam tests on multiple different testing facilities. Some carried out pendulum tests where the specimens, rotated along the longitudinal axis and mounted to a special support plate, were hit by a pendulous mass of up to $50 \mathrm{~kg}$, e.g. Máca et al. [1]. Other researchers like Fujikake and colleagues [2, 3, 4] carried out tests in servo-controlled rapid loading machines. After an initial acceleration period, these machines applied a constant loading velocity to the RC beams. Some specimens were additionally reinforced by stirrup reinforcement with varying spacing. The result was a relatively fine crack pattern redounding in diagonal tension failure, shear compression failure or flexural failure. The results of the strain gauge measurements taken on the longitudinal reinforcement as well as the stirrups that are mentioned in [2] were neither shown nor compared to the impact velocity.

The impact tests reported in this paper were carried out in the drop tower of the Otto Mohr Laboratory at TU Dresden [5]. The beams were simply supported by two rollers and minimally held down by unprestressed bolts and a transverse steel girder. The impactor hit the specimen after sliding through a steel pipe with a length of about $11 \mathrm{~m}$. Compressed

\footnotetext{
*Corresponding author: lena.leicht@tu-dresden.de
} 
air accelerated the impactor in addition to the fall energy. The final velocity of the impactor was derived from the signals of two light barriers at the end of the pipe. Thus, the loading conditions of the tests presented in $[2,3,4]$ differed strongly from the loading in this study. Here, a hard and short impact [6] was applied to the beams, whereas in [2, 3, 4] a slower and constant velocity impact strained the specimens.

There are three main effects resulting from the impact loading that lead to steel strains over the whole length of the reinforcement bar. The first effect is the bending of the beam due to the impact force. Apart from that, shear and longitudinal waves start propagating within the beam after the impact. The longitudinal waves are the most relevant ones as for this type of wave, the direction of the wave propagation is equal to the direction of particle movement leading to stresses and strains within the specimen and thus over the length of the reinforcement bar. These waves do not only travel vertically but also spread in the horizontal direction [7]. The vertically travelling part of the longitudinal waves is reflected as a tensile wave at the free bottom surface of the beam. The combination of high shear forces due to the locally acting impact force and the reflected wave is responsible for the typical cone failure of the beams. This combined shear and scabbing failure mode leads to strain concentrations within the steel in the region where the inclined cracks of the typical punching cone open. Therefore, the formation of the punching cone is the third reason for strains in the reinforcement.

This study aims to analyse the cracking process and to investigate the influence of the impactor energy on the steel strains and resulting strain rates in drop tower beam tests with high loading velocities and low impactor masses.

\section{Experimental study}

\subsection{Geometry of the girders and measurement}

The tested beams were 1,450 $\mathrm{mm}$ long, $280 \mathrm{~mm}$ high and $130 \mathrm{~mm}$ wide. The diameter of the longitudinal reinforcement was $8 \mathrm{~mm}$ and the beams were not additionally reinforced by stirrup reinforcement to guarantee comparativeness to the impact tests on plates carried out at TU Dresden $[8,9,10]$. The bottom layer consisted of three reinforcement bars, while only two reinforcement bars formed the upper layer. These were held in place by transport handles. The concrete cover of the lower reinforcement layer was $10 \mathrm{~mm}$. This layer was instrumented with one semiconductor strain gauge (SG) in the middle of each of the three reinforcement bars. While the two outer bars were completely bonded to the surrounding concrete, the middle of the inner reinforcement bar was unbonded over a length of $100 \mathrm{~mm}$.

The concrete strength was tested after 28 days on four cylinders, the individual values were 49.3 MPa, 51.7 MPa, 51.9 MPa, and 54.5 MPa, respectively. The modulus of elasticity ranged between $33,800 \mathrm{MPa}$ and $37,300 \mathrm{MPa}$.

\subsection{Impactor geometry and speed}

Two impactors with different lengths $-380 \mathrm{~mm}$ and $250 \mathrm{~mm}$-and thus different weights were used. The impactors had a diameter of $100 \mathrm{~mm}$ and were made of steel. Their hitting surface was flat. An overview of the experiments carried out with the different impactors and varying loading pressures can be seen in Tab. 1. Per configuration, one beam was tested. The names of the specimens consist of the impactor length in millimetre and the loading pressure in bar. 
Table 1. Experimental matrix.

\begin{tabular}{|c|c|c|c|c|c|c|}
\hline & \multicolumn{6}{|c|}{ Loading pressure [bar] } \\
\hline & 0 & 0.2 & 0.4 & 0.6 & 0.8 & 1.0 \\
\hline 馬 & B380_0.0 & B380_0.2 & B380_0.4 & B380_0.6 & B380_0.8 & B380_1.0 \\
\hline 吾哭 & B250_0.0 & B250_0.2 & B250_0.4 & B250_0.6 & - & - \\
\hline
\end{tabular}

\section{Results}

\subsection{Impact energy}

The impact energy is a good criterion to compare different tests because it takes the impactor velocity as well as its weight into account. The only element which cannot be taken into account in the energy-based analysis is the wave length of the impact wave which depends on the length of the impactor. As wave propagation cannot really be studied with three strain gages at the same position regarding the length of the beam, this is not considered a disadvantage. The impact energy is defined as the kinetic energy of the impactor calculated from its mass and its velocity.

$$
E_{\mathrm{Imp}}=0.5 \cdot m_{\mathrm{I}} \cdot v_{\mathrm{I}}^{2}
$$

where: $\quad E_{\mathrm{imp}}-$ impact energy

$m_{\mathrm{I}}$ - impactor mass

$v_{\mathrm{I}}$ - impactor velocity

\subsection{General behaviour of the beams}

The overall failure mode of all tested beams was the typical formation of a punching cone (see. Fig. 1 and 2) which differed strongly from the failure modes reported in [2, 3, 4], where cracks more distributed than in the present study were observed, which led to a more ductile failure.

Regarding the influence on the stress in the lower reinforcement layer, the beam can be split in three different areas after the impact, which can be seen in Fig. 1 a). No. 1 is the mentioned punching cone which can be as high as the beam or lower. Area number 2 is characterized by the spalling off of the concrete cover due to the untying of the cone from the rest of the beam. Lastly, area 3 is marked by the end anchorage of the reinforcement bars, which is uncritical for small cones which guarantee large anchorage lengths but can become critical for large cones with small anchorage lengths. However, predicting the width of the punching cone is not possible as it does not clearly depend on the loading velocity. On top of that, the cones sometimes were asymmetric, especially for small loading pressures, see Fig. 1 a) and 2 a).

The cracking process during the impact was evaluated with the help of high speed camera videos that recorded at the rate of 10,000 frames/s. For almost all beams, cracking initiated with the bending crack in the middle of the beam. These cracks, which became about half as long as the beam's height, usually arose between $0.2 \mathrm{~ms}$ and $0.3 \mathrm{~ms}$ after the impact (e.g. B380_0.0, B380_0.2, B250_0.0, B250_0.4, and B250_0.6). Thereafter started the formation of the punching cone. There were tests where the formation of the cone started with inclined cracks from underneath the beam (e.g. B380_0.0, B380_0.4, and B250_0.0). In these 
specimens, the cone started to form from $0.4 \mathrm{~ms}$ to $0.6 \mathrm{~ms}$ after the impact. In the instances where the cracks of the punching cone were completely visible from one camera frame to the next, the cracks already formed from $0.3 \mathrm{~ms}$ to $0.4 \mathrm{~ms}$ after the impact (e.g. B380_0.2, B250_0.4, and B250_0.6). After the cone was separated from the rest of the cross section, it was accelerated leading to the spalling off of the concrete cover in area 2.

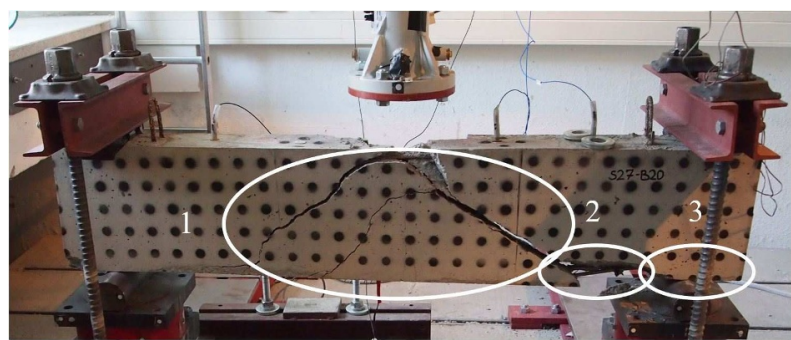

a) B380_0.0 (tested at 0 bar with the resulting impactor velocity of $13.4 \mathrm{~m} / \mathrm{s}$ )

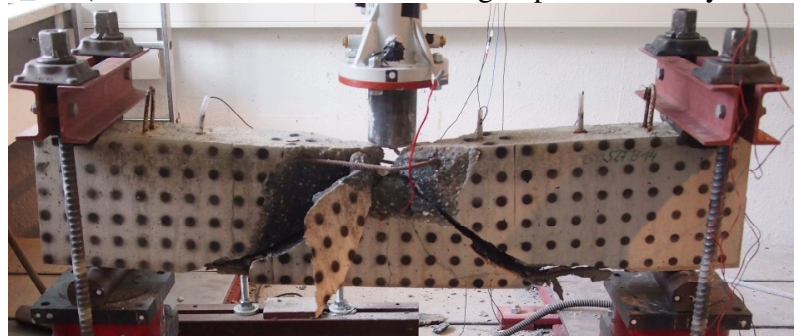

b) B380_0.4 (tested at 0.4 bar with the resulting impactor velocity of $19.8 \mathrm{~m} / \mathrm{s}$ )

Fig. 1. Punching cones for different loading velocities after impact with the $380 \mathrm{~mm}$ long impactor (weight: $21.66 \mathrm{~kg}$ )

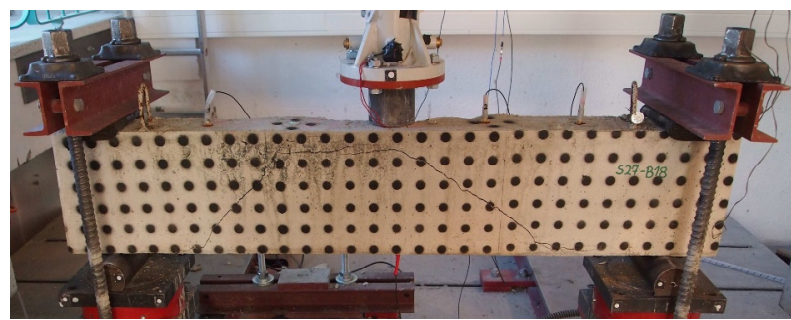

a) B250_0.0 (tested at 0 bar with the resulting impactor velocity of $12.4 \mathrm{~m} / \mathrm{s}$ )

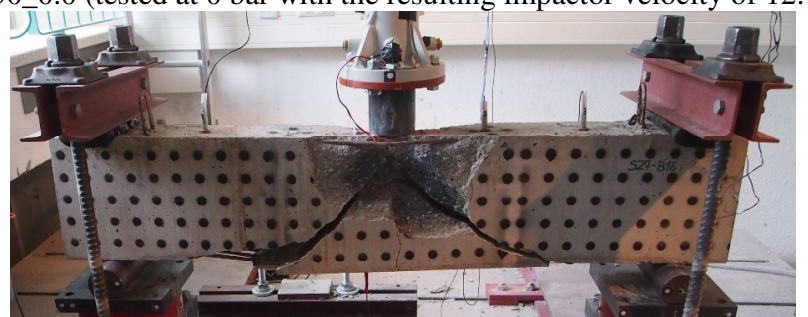

b) B250_0.4 (tested at 0.4 bar with the resulting impactor velocity of $22.5 \mathrm{~m} / \mathrm{s}$ )

Fig. 2. Punching cones for different loading velocities after impact with the $250 \mathrm{~mm}$ long impactor (weight: $14.22 \mathrm{~kg}$ )

By contrast, the time it took for the wave to pass through the beam in the vertical direction can be estimated to only $0.07 \mathrm{~ms}$ (see eq. $2[6,11]$ ). After these $0.07 \mathrm{~ms}$, the compressive wave was reflected as a tensile wave from the free bottom surface of the beam. As the initial wave travelled about four times through the cross section as a tensile and compressive pulse 
until the inclined cracking of the punching cone initiated, the waves interfered strongly in the area where the cracking occurs. This not only affected the waves travelling through the cross section, but the cracks also impeded the wave propagation as tensile stresses cannot be transferred via unreinforced, cracked regions.

$$
t_{\mathrm{c}}=h_{\mathrm{c}} / c_{\mathrm{c}}=280 \mathrm{~mm} / 4,112 \mathrm{~m} / \mathrm{s}=0.07 \mathrm{~ms}
$$

where: $t_{\mathrm{c}}$ - time it takes for the wave to travel the beam's height

$h_{\mathrm{c}}-$ height of the beam

$c_{\mathrm{c}}$ - wave propagation velocity of concrete

Towards the end of the test, the concrete in the upper region of the beam spalled off in the transverse direction. The cracks leading to this spalling were visible between $2.6 \mathrm{~ms}$ and $9.9 \mathrm{~ms}$ after the impact. The higher the impactor velocity and accordingly the impact energy was, the more damage happened in this area. This is well visualized by plotting the burst mass over the kinetic energy of the impactor (see Fig. 3). The burst mass is the sum of all fragments that fell off the beam and which were weighted after the test. The top of the punching cone interfered with the area of lateral concrete spalling leading to a smeared damage consisting of the shear failure and lateral spalling on the upper side of the beam (see Fig. $1 \mathrm{~b}$ ) and $2 \mathrm{~b}$ )). It is interesting that this lateral spalling did not occur right after the impact due to the lateral spreading of the impact force, but happened simultaneously to the cone untying from the rest of the concrete beam. This is why it did not happen when the acceleration force of the cone is small as is the case for specimen B250_0.0 (see Fig. 2 a)). The cone's acceleration thus depends on the impact energy. Moreover, the impact most likely formed a three-dimensional cone like in the case of the plates $[8,9,10]$. That means that the cone gained width over both the length and the width of the concrete beam. This favoured the bursting at the top of the beam due to inclined cracks resulting from the formation of a three-dimensional cone.

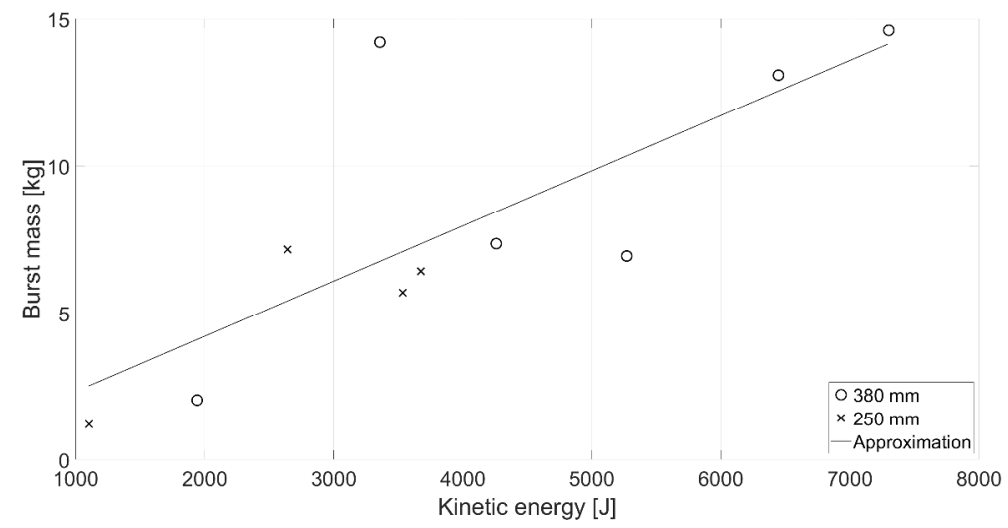

Fig. 3. Relationship between energy and burst mass

\subsection{Influences on the maximum strains}

The time lags between impact and maximum strains (Fig. 5) on the one hand, and the maximum strains (Fig. 6) and the maximum strain rates (Fig. 7), each compared to the impact energy. On the other hand, they were evaluated for the bonded and the unbonded strain gauges separately. In the case of strain gauges 1 and 3, the approximation is calculated from the absolute measured maxima of each test but the maximum of each gauge is displayed. The maximum strains measured by the unbonded strain gauges (SG no. 2) were between 4 and 7 
times higher than the ones recorded by the bonded strain gauges. That is probably the reason why eight of the ten unbonded gauges failed in the peak region before reaching peak II. The only two specimens where they recorded over the whole test duration were the beams B380_0.4 and B250_0.6. Fig. 4 shows the strains measured in B380_0.4. The reason for the high peak measured by the unbonded strain gauges was the bending crack in the middle. In all experiments, bending of the beams caused only one crack in the centre which opened quickly and led to a high strain concentration and thus plasticisation in the reinforcement at the position of the crack. The peak strain thus can only be measured if the strain gauge was applied at the exact position where the bending crack appeared. This means of course, that it was very unlikely to measure the peak with the bonded strain gauges as they would need to be located exactly where the crack opens. However, it was possible to measure the peak strain with the unbonded strain gauge as it measured an average strain in the region where the bending crack appeared and because the strains are almost constant over the unbonded length until necking starts. The only strains occurring on a local level before necking are the yielding strains [12]. As the strains measured by the strain gauge in the middle never reached the necking point, the assumption that the strains are approximately constant over the whole unbonded length is valid.

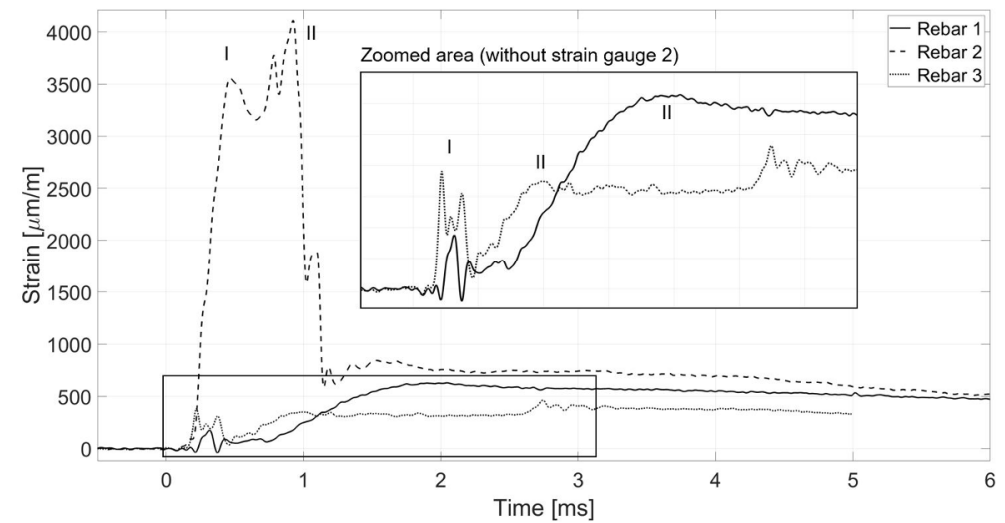

Fig. 4. Strains measured by the three strain gauges of B380_0.4. SG 1 and 3 (solid and dotted line) were bonded whereas SG 2 (dashed line) was unbonded. The impact happened at time ' 0 '.

The two bonded strain gauges generally measured two distinct peaks I and II (see Fig. 4), which can be seen in the measurement of strain gauge 2 as well but they were not as obvious due to the generally higher strains. The first strains measured in all gauges started with the formation of the bending crack between $0.2 \mathrm{~ms}$ and $0.3 \mathrm{~ms}$ after the impact. The first peak I was measured about $0.5 \mathrm{~ms}$ after the impact which is right before the formation of the punching cone. This peak was a little delayed for the second strain gauge compared to the other two because the strains needed to be transferred from the position where the concrete cracked to the ends of the pipe and again to the middle of the reinforcement bar to the position of the strain gauge. From the time the punching cone arose and untied from the cross section, the strains decreased. The probable reason is that the bending radius in the untying part of the section increased leading to lower bending strains.

The time when the second peak II was reached, which is higher than the first one for almost all beams, is plotted against the impactor velocity in Fig. 5. The only beams where the second peak II was not existent, and therefore lower than the first one, were beam B380_1.0, which was completely destroyed and therefore hard to evaluate, and beam B250_0.0, where the cone did not separate from the rest of the section (see Fig. 2 a) and which thus behaved differently from the other beams. Therefore, the measurements of beams B380_1.0 and B250_0.0 (marked with a circle in Fig. 5-7) were not included in the analysis. 
However, this points out that this global maximum of the bonded strain gauges was influenced by the untying of the cone. Furthermore, the time of the second peak II was not equal for all tests and therefore depended on the loading velocity. There seems to be a long time lag for low impactor velocities which decreases with increasing impactor velocities. This trend can be observed for strain gauges 1 and 3 (black) as well as for strain gauge 2 (red), even though-as already explained-the evaluation of the time lag for the strain gauges no. 2 was difficult since most of them were destroyed before the second maximum.

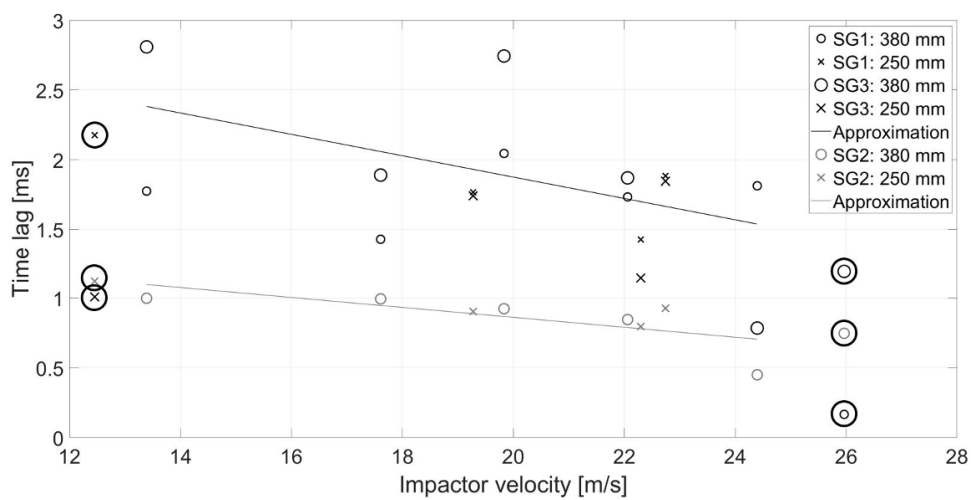

Fig. 5. Time lag between impact and maximum strain. Approximation for SG 1 and 3 (black) and SG 2 (grey). Data points of beams B380_1.0 and B250_0.0 (circled) were excluded in the analysis.

A rise in the impact energy affected the maximum strains in the centre, which were equal to peak II in Fig. 4, only slightly (see Fig. 6). The strains measured by the unbonded strain gauges slightly decreased, whereas the maximum strains of the bonded strain gauges slightly increased. There seems to be a maximum between 3,000 J and 4,000 J for the bonded strain gauges. However, the strong scattering of the values makes the relation weak. The relation between the maximum strain and kinetic energy for the unbonded strain gauges is not very robust either because most strain gauges failed in the peak region before peak II was even reached. This leads to an uncertainty concerning the reliability of the measured data. Additionally, it is unclear whether the relationship between the strains and resistance of the strain gauges remained linear at strains as high as $4,800 \mu \mathrm{m} / \mathrm{m}$, although the manufacturer claims the dynamic tensile strain limit to be $5,000 \mu \mathrm{m} / \mathrm{m}$.

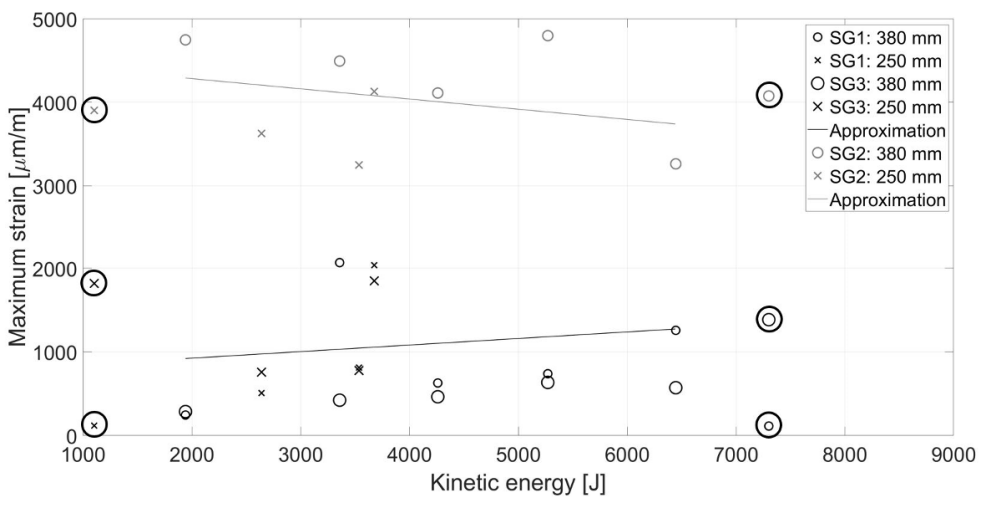

Fig. 6. Relationship between energy and maximum strain. Approximation for SG 1 and 3 (black) and SG 2 (grey). Data points of beams B380_1.0 and B250_0.0 (circled) were excluded in the analysis. 
A special aspect of certain strain measurements for strain gauges 1 and 3 should be pointed out: it seems that there remained a residual strain after the second peak II (compare Fig. 4). There are two possible explanations for this behaviour.

(1) The steel deformed above its yield strain and a plastic strain remained after the impact. It is unrealistic, however, that the steel deformed plastically if it was not stressed above the yield strength as is the case for all bonded strain gauges with the maximum strain of about $2000 \mu \mathrm{m} / \mathrm{m}$ (see Fig.6). The theoretical yield strain of steel is $2000 \mu \mathrm{m} / \mathrm{m}$. Nevertheless, it is unclear whether the peak of the bonded strain gauges is measured correctly as the strains were averaged over a gauge length of $2.6 \mathrm{~mm}$.

(2) A second option is that the measured residual strains were not really plastic strains but result from the fact that the mass of the untied punching cone is solely held by the reinforcement bars. This would also explain, why the residual strains in all three reinforcement bars are similar.

Another remarkable fact is that there are considerable differences between the measurements of strain gauge 1 and 3 in some cases. This could be due to an eccentric impact. Further experiments and numerical studies are planned in the near future (see Section 4.2) in order to analyse these currently insufficiently clarified phenomena in greater depth.

\subsection{Influences on the maximum strain rates}

The strain rates were directly derived from the strains. To smoothen the strain signal before calculating the strain rates, a strain vector consisting of the medians of 250 single strain values for each element of the strain rate vector was used. The maximum strain rate of each test is plotted in Fig. 7. To control the derivation result, the signals obtained from integration of the strain rates were compared to the measured strains. In fact, these graphs matched the real strain signals well.

Fig. 7 shows that the strain rates had a similar relation to the impact energy as the strains (Fig. 6). That means that the strain rates as well as the strains were almost independent of the kinetic energy. Excluded from the analysis were again beam B380_1.0 and B250_0.0 (marked in Fig. 7). As for the strains, the strain rates of the unbonded strain gauges were multiple times higher than for the bonded ones. This was due to the fact that the maximum strains were a lot higher for the unbonded strain gauges and the two values were occurring almost simultaneously.

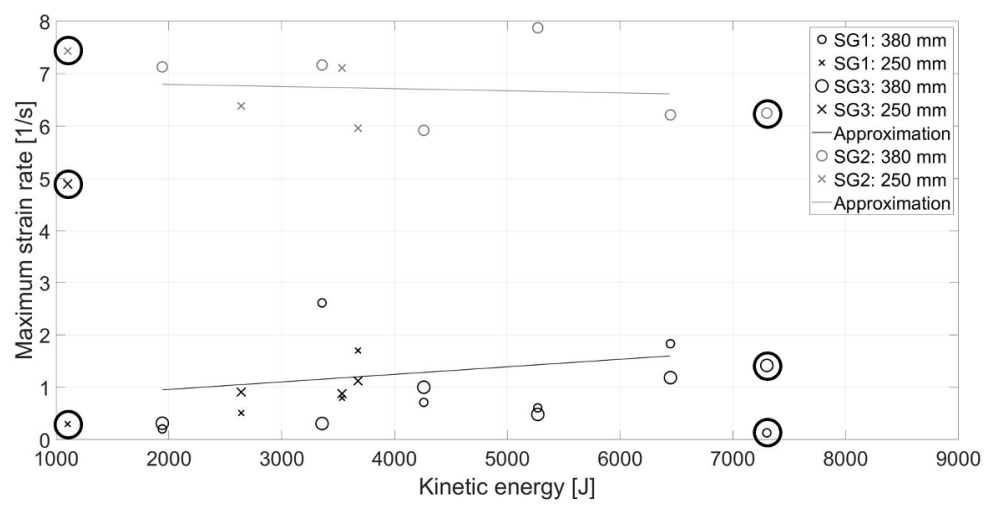

Fig. 7. Relationship between energy and maximum strain rate. Approximation for SG 1 and 3 (black) and SG 2 (grey). Data points of beams B380_1.0 and B250_0.0 (circled) were excluded in the analysis.

In about half of the cases, the maximum strain rate for gauges 1 and 3 occurred in the approach of the first peak of the strains whereas it was measured in the approach of the second 
peak for the other half of the strain gauges. This enforces the hypothesis that the strain rate was independent of the loading velocity. For the unbonded strain gauges, the maximum strain rate was in all cases measured during the approach of the first peak.

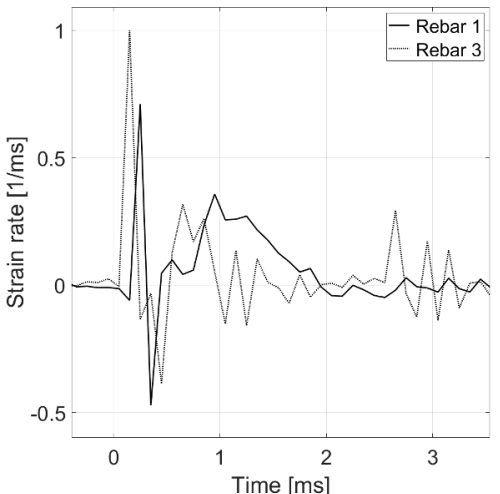

a) B380_0.4

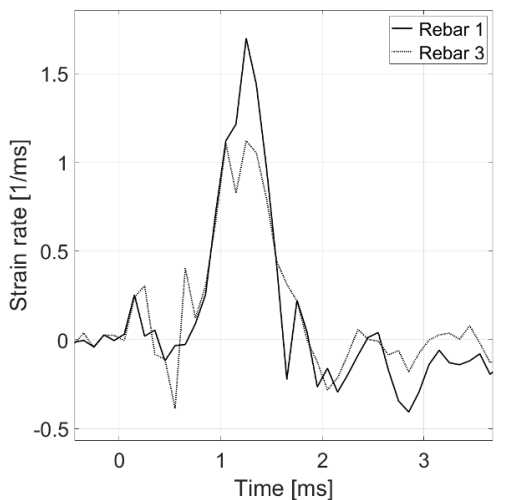

b) B250_0.4

Fig. 8. Comparison of strain rate

\section{Conclusions}

\subsection{Influences on the structural behaviour, maximum strains and strain rates}

The inclination of the punching cone depended only little on the impactor energy. However, the acceleration of the cone increased with increasing impact velocity leading to wider cracks. This is also the reason for higher burst masses at higher impact energies.

The steel strains in the middle of the beam were almost independent of the impactor energy. There were two peaks in the strain measurement, the first after about $0.5 \mathrm{~ms}$. This was the time when the punching cone started to form. The time lag to the second peak depended on the impactor velocity as it decreased with increasing impactor velocity. The maximum strain measured by the bonded strain gauges was approximately $2,000 \mu \mathrm{m} / \mathrm{m}$. However, the measured peaks vary strongly in some specimens. The strains measured by the unbonded strain gauges were multiple times higher because they captured the localized bending strains. They were as high as $4,800 \mu \mathrm{m} / \mathrm{m}$. However, it was hard to relate the measurements of the unbonded strain gauges to any other measured data as only two of them worked for the whole test duration.

The strain rates of the bonded strain gauges ranged between $0.31 / \mathrm{s}$ and $2.51 / \mathrm{s}$. As well as the strains, they did not really depend on the impact energy. The maximum strain rates of the unbonded strain gauges, by contrast, reached values between $5.91 / \mathrm{s}$ and $7.91 / \mathrm{s}$.

\subsection{Conclusions}

To confirm the findings, a more detailed study with different impactors and fine-tuned impact energies should be performed. The impactor velocity as well as the impactor mass and its nose geometry should be varied. In addition, plates could be used as pulse shapers to evaluate softer impact scenarios with more distributed crack patterns. The supports can be changed to fixed supports by applying a prestressing force to the bolts holding the beams down to vary the support conditions. After the test, the reinforcement could be pulled out and checked for local plasticisation which could also prove whether the residual strains in the bonded strain gauges were plastic or whether they resulted from holding the punching cone. 
Additionally, tests with multiple strain gauges over the length of the reinforcement bar will be performed to see differences between the strains and strain rates at different positions. It is likely that the strains measured close to the punching cone and therefore to wider cracks are higher than those measured in the middle of the beam. The multitude of strain gauges over the length of the reinforcement bar furthermore enables the analysis of bond stresses and wave propagation within the specimen. The fact that all strain gauges were at the same position in the present study makes it impossible to capture these effects and to decide whether the strains resulted from wave bending, wave propagation or the formation of the punching cone. To guarantee the measurement of plastic strains in the region of wide cracksin the middle of the beam and at the position of the punching cone-unbonded strain gauges can be applied.

Moreover, different beam geometries should be tested. The beam's height as well as thickness should be varied because they influence wave propagation and the shape of the punching cone. With a reduced thickness, the punching cone will be more two-dimensional, probably leading to lower burst masses. In addition, the reinforcement can be varied and stirrup reinforcement can be used.

To complete the experiments, a Finite Element Analysis will be conducted. This FEA gives insight into the specimens and allows finer evaluation of the reinforcement strains.

Lastly, small scale tests in the split Hopkinson pressure bar can be derived from the beam tests. This helps to conduct realistic bond tests based on the experimentally observed behaviour of the beams and the reinforcement within them.

The presented project was funded by the German Federal Ministry of Economic Affairs and Energy (BMWi, project no. 1501566) on the basis of a decision by the German Bundestag.

\section{References}

1. P. Máca, J. Zatloukal, R. Sovják, Structures under Shock and Impact XIII, 149-158 (2014).

2. A. Somraj, K. Fujikake, B. Li, International Journal of Protective Structures 4, 521543 (2013).

3. K. Fujikake, B. Li, S. Soeun, Journal of Structural Engineering 135, 938-950 (2009).

4. S. D. Adhikary, B. Li, K. Fujikake, International Journal of Protective Structures 6, 1, 81-111 (2015).

5. M. Just, M. Curbach, T. Kühn, M. Hering, Behavior of structural components during impact load conditions caused by tank collisions (aircraft fuel tanks): Phase 1A: Scale effects under impact loading (Final report of the project 1501438 funded by BMWi) (2016), doi: https://doi.org/10.2314/GBV:868615218.

6. T. Kühn, D. Schmidt, T. Hänztschel, O. Million, A. Stolz, M. Curbach, K. Thoma, Bautechnik 93, 717-724 (2016).

7. M. A. Meyers, Dynamic behavior of materials, (University of California, San Diego, 1994).

8. M. Hering, F. Bracklow, T. Kühn, M. Curbach, Struct. Concr., 21, 587-598 (2020).

9. T. Kühn, M. Curbach, Proceedings of DYMAT, 7-11 (2015).

10. M. Hering, T. Kühn, M. Curbach, U. Häußler-Combe, Proceedings of the fib Symposium (2016).

11. L. E. Kinsler, A. R. Frey, A. B. Coppens, J. V. Sanders. Fundamentals of Acoustics (Wiley, 2000).

12. F. Hahn, Werkstofftechnik-Praktikum: Werkstoffe prüfen und verstehen (Fachbuchverlag Leipzig im Carl Hanser Verlag, 2015). 